\title{
Scattering on plasmonic nanostructures arrays modeled with a surface integral formulation
}

\author{
Benjamin Gallinet*, Olivier J.F. Martin \\ Nanophotonics and Metrology Laboratory, Swiss Federal Institute of Technology Lausanne (EPFL), \\ 1015 Lausanne, Switzerland
}

Received 28 January 2010; received in revised form 26 May 2010; accepted 31 May 2010

Available online 8 June 2010

\begin{abstract}
The surface integral formulation is a flexible, multiscale and accurate tool to simulate light scattering on nanostructures. Its generalization to periodic arrays is introduced in this paper. The general electromagnetic scattering problem is reduced to a discretizated model using the Method of Moments on the surface of the scatterers in the unit cell. The study of the resonances of an array of bowtie antennas illustrates the main features of the method. When placed into an array, the bowtie antennas show additional resonances compared to those of an individual antenna. Using the surface integral formulation, we are able to investigate both nearfield and far-field properties of these resonances, with a high level of accuracy.
\end{abstract}

(C) 2010 Elsevier B.V. All rights reserved.

Keywords: Electromagnetic scattering; Periodic systems; Plasmonic antennas; Resonances

\section{Introduction}

Single metal nanoparticles exhibit characteristic localized surface plasmon modes which depend on different parameters such as their size, composition, shape and surrounding medium [1]. When placed into periodic arrays, their resonant properties are strongly affected, since the resonances of the individual nanoparticles couple with the periodicity. This observation has paved the road to the design of novel materials with tailored optical properties: negative index of refraction metamaterials [2], photonic band-gap materials [3], plasmon-enhanced aperture [4,5] and subwavelength optical structures [6,7]. Plasmonic systems involve high index contrasts and strong electromagnetic resonances with high field variations, two conditions

\footnotetext{
* Corresponding author. Tel.: +41216936843.

E-mail address: benjamin.gallinet@epfl.ch (B. Gallinet).
}

very challenging for numerical modeling. There is therefore an increasing demand for a modeling tool accurate even in these extreme conditions. A wide variety of methods is available for modeling the interaction of electromagnetic radiation with matter: couple-mode theory [8], finite-difference time-domain [9], plane-wave expansion [10], finite elements method [11] (FEM). The FEM is proven very accurate, flexible to handle realistic structures [12], and produces sparse matrices efficiently solved with specific algorithms. However, it suffers from the need to impose boundary conditions at the edges of the computation window [11].

State of the art optical measurement techniques offer the possibility to study optical far-field properties as well as to give a deep insight into the corresponding near-field physics [13]. Integral equations are multiscale methods that only discretize the scatterer, but the matrices describing the scattering system are dense and require high memory for large systems $[14,15]$. With surface integral equation (SIE) methods $[16,17]$ only 
the scatterer surface is discretized. Since SIE methods also generate dense matrices, the fact that they scale with only the second power of the lateral dimension of homogeneous scatterers greatly improves their efficiency. For non-homogeneous scatterers hybrid FiniteElement-Boundary Integral methods are proven efficient and very popular for microwaves $[18,11]$. In the SIE developed in Ref. [17] the Method of Moments (MoM) [11] has been successfully applied to plasmonic scatterers and guarantees a high precision and flexibility of the discretization. In Ref. [19], a MoM-based SIE method is applied to periodic nanostructures from microwave regime studies (see e.g. [20,21]). This SIE formulation can be derived and discretized for an arbitrary number of materials and can handle a wide variety of scatterers (e.g. composite or embedded in multilayered media). The purpose of this paper is to focus on its application to plasmonics.

Section 2 gives an overview of the SIE formulation for periodic systems (for a more detailed approach see Ref. [19]). In Section 3, as an illustration of one of the possible applications of this method, the case of an array of realistic gold bowtie nanoantennas is investigated. Their complex shape and metallic properties make them a good example of the versatility and accuracy of the method. A comparative study of light scattering at normal incidence on a single antenna and on an infinite array of antennas illustrates different resonance properties.

\section{Surface integral formulation for electromagnetic scattering on periodic structures}

The purpose of this section is to give an overview of the numerical method. The simulation of electromagnetic scattering is reduced to the computation of equivalent surface currents on the boundaries between the different media in the unit cell. These currents are the solution of the Electric Field Integral Equation (EFIE) and the Magnetic Field Integral Equation (MFIE). The first part of this section generalizes this formulation to periodic systems, and shows that the EFIE and MFIE are restricted to the unit cell with periodic boundary conditions. The EFIE and MFIE involve the pseudo-periodic dyadic Green's function, whose evaluation can be accelerated with Ewald's method [19,22-24]. The MoM is used to discretize and solve the integral equations for the equivalent surface currents. The scattered electric and magnetic fields are calculated from the equivalent surface currents during post-processing at any point of space. The singularity subtraction technique for the Green's function provides a high precision of the surface currents and enables the field sampling arbitrarily close to the surface.

\subsection{Electric and magnetic integral equations}

The three-dimensional space is divided into $N$ different regions $V_{n}, n=1, \ldots N$ with dielectric permittivity $\epsilon_{n}$ and magnetic permeability $\mu_{n}$ (Fig. 1). A harmonic time-dependence of the fields $\mathbf{U}(\mathbf{r}, t)=$ $\mathbf{U}_{0}(\mathbf{r}) e^{-i \omega t}$ is assumed throughout this paper. The electric field $\mathbf{E}$ in each region must satisfy the equation:

$\nabla \times \nabla \times \mathbf{E}(\mathbf{r})-k_{n}^{2} \mathbf{E}(\mathbf{r})=i \omega \mu_{n} \mathbf{j}(\mathbf{r}), \mathbf{r} \in V_{n}$,

where $k_{n}^{2}=\omega^{2} \epsilon_{n} \mu_{n}$ is the wavenumber for electromagnetic waves in region $n$ and $\mathbf{j}$ denotes the volume current density. A dyadic Green's function $\underline{\underline{G}}_{n}$ for region $V_{n}$ is introduced:

$\nabla \times \nabla \times \underline{\underline{G}}_{n}\left(\mathbf{r}, \mathbf{r}^{\prime}\right)-k_{n}^{2} \underline{\underline{G}}_{n}\left(\mathbf{r}, \mathbf{r}^{\prime}\right)=\underline{\underline{1}} \delta\left(\mathbf{r}-\mathbf{r}^{\prime}\right)$.

Combining Eqs. (1) and (2), integrating over $\mathbf{r}$ and applying Green-Gauss theorem, leads to a surface integral on the boundary $\partial V_{n}$ of region $V_{n}$ :

$$
\begin{array}{rl}
\int_{\partial V_{n}} & d S \hat{\mathbf{n}}_{n}(\mathbf{r}) \\
& \cdot\left([\nabla \times \mathbf{E}(\mathbf{r})] \times \underline{\underline{G}}_{n}\left(\mathbf{r}, \mathbf{r}^{\prime}\right)+\mathbf{E}(\mathbf{r})\right. \\
& \left.\times\left[\nabla \times \underline{\underline{G}}_{n}\left(\mathbf{r}, \mathbf{r}^{\prime}\right)\right]\right) \\
= & \mathbf{E}_{n}^{\text {inc }}\left(\mathbf{r}^{\prime}\right)- \begin{cases}\mathbf{E}\left(\mathbf{r}^{\prime}\right): & \mathbf{r}^{\prime} \in V_{n} \\
0: & \text { otherwise }\end{cases}
\end{array}
$$

where $\hat{\mathbf{n}}_{n}$ is the outward oriented normal vector on the boundary $\partial V_{n}$. Taking the second case in the right-hand side of Eq. (3), the continuity of the tangential component of the fields $\mathbf{E}$ and $\mathbf{H}$ allows one to take the limit $\mathbf{r}^{\prime} \rightarrow \partial V_{n}$. Hence, introducing the equivalent surface

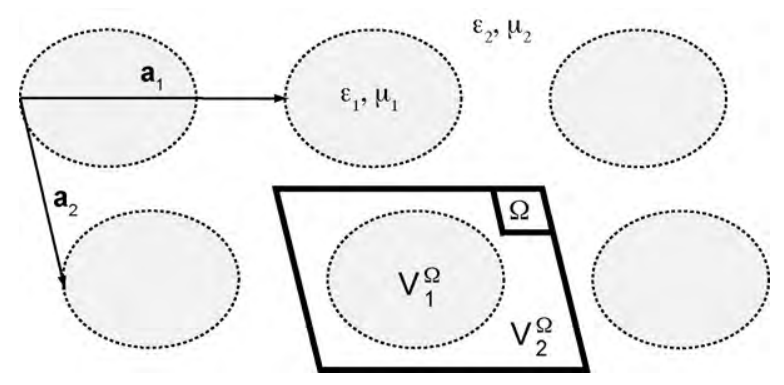

Fig. 1. Space division into regions $V_{n}$ with dielectric permittivity $\epsilon_{n}$ and magnetic permeability $\mu_{n}$. The system has the symmetry of a twodimensional lattice with unit cell $\Omega$ and primitive lattice vectors $\mathbf{a}_{1}$ and $\mathbf{a}_{2}$. The regions $V_{n}^{\Omega}$ are the intersection of the regions $V_{n}$ with the unit cell. 
current densities $\mathbf{J}_{n}=\hat{\mathbf{n}}_{n} \times \mathbf{H}$ and $\mathbf{M}_{n}=-\hat{\mathbf{n}}_{n} \times \mathbf{E}$ defined on $\partial V_{n}$, one can write the Electric Field Integral Equation (EFIE):

$$
\begin{gathered}
\left(\begin{array}{c}
i \omega \mu_{n} \int_{\partial V_{n}} d S^{\prime} \underline{\underline{G}}_{n}\left(\mathbf{r}, \mathbf{r}^{\prime}\right) \cdot \mathbf{J}_{n}\left(\mathbf{r}^{\prime}\right) \\
+\int_{\partial V_{n}} d S^{\prime}\left[\nabla^{\prime} \times \underline{\underline{G}}_{n}\left(\mathbf{r}, \mathbf{r}^{\prime}\right)\right] \cdot \mathbf{M}_{n}\left(\mathbf{r}^{\prime}\right)
\end{array}\right)_{\tan } \\
=\left(\mathbf{E}_{n}^{\operatorname{inc}}(\mathbf{r})\right)_{\tan }, \mathbf{r} \rightarrow \partial V_{n}, \mathbf{r} \notin V_{n},
\end{gathered}
$$

where the subscript tan denotes the tangential component of the fields and

$\mathbf{E}_{n}^{\mathrm{inc}}\left(\mathbf{r}^{\prime}\right)=i \omega \mu_{n} \int_{V_{n}} d V \underline{\underline{G}}_{n}\left(\mathbf{r}^{\prime}, \mathbf{r}\right) \cdot \mathbf{j}(\mathbf{r})$

is the incident electric field generated by source currents j in region $V_{n}$.

Although the method can be applied for lattices in 1, 2 or 3 dimensions, we shall now focus on the most common physical situation and assume that the regions $V_{n}$ carry the symmetry of a two-dimensional lattice (Fig. 1). A lattice translation vector $\mathbf{t}$ is a linear combination $\mathbf{t}=\sum_{i} c_{i} \mathbf{a}_{i}$ with $c_{i} \in \mathbb{Z}$ and $\mathbf{a}_{i}, i=1,2$ the primitive lattice vectors. The symmetry assumption is then written as $\mathbf{r} \in V_{n} \Rightarrow \mathbf{r}+\mathbf{t} \in V_{n}, \forall \mathbf{t}$. The unit cell is called $\Omega$. The irreducible representations of the translation group are associated to a Bloch wavevector $\mathbf{k}$ in the first Brillouin zone. Bloch waves $\mathbf{U}_{\mathbf{k}}$ are characterized by their transformation properties under translations $\mathbf{t}$, also known as periodic boundary conditions:

$\mathbf{U}_{\mathbf{k}}(\mathbf{r}-\mathbf{t})=e^{-i \mathbf{k} \cdot \mathbf{t}} \mathbf{U}_{\mathbf{k}}(\mathbf{r})$.

The periodic dyadic Green's function $\underline{G}_{n \mathbf{k}}$ is the projection of $\underline{G}_{n}$ onto the Bloch component $\mathbf{k}$ :

$\underline{\underline{G}}_{n, \mathbf{k}}\left(\mathbf{r}, \mathbf{r}^{\prime}\right)=\sum_{\mathbf{t}} e^{i \mathbf{k} \cdot \mathbf{t}} \underline{\underline{G}}_{n}\left(\mathbf{r}-\mathbf{t}, \mathbf{r}^{\prime}\right)$

It satisfies $\underline{\underline{G}}_{n, \mathbf{k}}\left(\mathbf{r}-\mathbf{t}, \mathbf{r}^{\prime}\right)=e^{-i \mathbf{k} \cdot \mathbf{t}} \underline{\underline{G}}_{n, \mathbf{k}}\left(\mathbf{r}, \mathbf{r}^{\prime}\right)$ and $\underline{\underline{G}}_{n, \mathbf{k}}$ $\left(\mathbf{r}, \mathbf{r}^{\prime}\right)^{\dagger}=\underline{\underline{G}}_{n, \mathbf{k}}\left(\mathbf{r}^{\prime}, \mathbf{r}\right)$ where $\underline{\underline{G}}_{n, \mathbf{k}}\left(\mathbf{r}, \mathbf{r}^{\prime}\right)^{\dagger}$ is the conjugate transposed of $\underline{\underline{G}}_{n, \mathbf{k}}\left(\mathbf{r}, \mathbf{r}^{\prime}\right)$. The periodicity yields a reduction of the EFIE to $\partial V_{n} \cap \Omega \equiv \partial V_{n}^{\Omega}$ (Fig. 1):

$$
\left(\begin{array}{c}
i \omega \mu_{n} \int_{\partial V_{n}^{\Omega}} d S^{\prime} \underline{\underline{G}}_{n, \mathbf{k}}\left(\mathbf{r}, \mathbf{r}^{\prime}\right) \cdot \mathbf{J}_{n, \mathbf{k}}\left(\mathbf{r}^{\prime}\right) \\
+\int_{\partial V_{n}^{\Omega}} d S^{\prime}\left[\nabla^{\prime} \times \underline{\underline{G}}_{n, \mathbf{k}}\left(\mathbf{r}, \mathbf{r}^{\prime}\right)\right] \cdot \mathbf{M}_{n, \mathbf{k}}\left(\mathbf{r}^{\prime}\right)
\end{array}\right)_{\tan }
$$

Starting from the magnetic field equivalent of Eq. (1) and defining $\mathbf{H}_{n}^{\text {inc }}$ as the incident magnetic field equivalent to Eq. (5), we can similarly derive the MFIE in the unit cell:

$$
\begin{aligned}
& \left(\begin{array}{c}
i \omega \epsilon_{n} \int_{\partial V_{n}^{\Omega}} d S^{\prime} \underline{\underline{G}}_{n, \mathbf{k}}\left(\mathbf{r}, \mathbf{r}^{\prime}\right) \cdot \mathbf{M}_{n, \mathbf{k}}\left(\mathbf{r}^{\prime}\right) \\
-\int_{\partial V_{n}^{\Omega}} d S^{\prime}\left[\nabla^{\prime} \times \underline{\underline{G}}_{n, \mathbf{k}}\left(\mathbf{r}, \mathbf{r}^{\prime}\right)\right] \cdot \mathbf{J}_{n, \mathbf{k}}\left(\mathbf{r}^{\prime}\right)
\end{array}\right)_{\tan } \\
& =\left(\mathbf{H}_{n, \mathbf{k}}^{\mathrm{inc}}(\mathbf{r})\right)_{\tan } .
\end{aligned}
$$

The fields $\mathbf{J}_{n, \mathbf{k}}, \mathbf{M}_{n, \mathbf{k}}, \mathbf{E}_{n, \mathbf{k}}^{\mathrm{inc}}$ and $\mathbf{H}_{n, \mathbf{k}}^{\mathrm{inc}}$ denote the projection of the fields $\mathbf{J}_{n}, \mathbf{M}_{n}, \mathbf{E}_{n}^{\text {inc }}$ and $\mathbf{H}_{n}^{\text {inc }}$ to Bloch waves with corresponding wavevector $\mathbf{k}$ and satisfying the periodic boundary conditions (6). If the source currents $\mathbf{j}$ do not satisfy these conditions, they should be expressed as a linear combination of Bloch waves. The total problem should then be decomposed and solved independently for each incident Bloch wave condition. In this paper, the surfaces $\partial V_{n}^{\Omega}$ do not touch the boundaries of the unit cell. For simplicity, the indexes $\mathbf{k}$ and $\Omega$ are omitted in the following.

\subsection{Solution by Method of Moments}

A technique for solving the EFIE (8) and the MFIE (9) is the Method of Moments (MoM). A two regions problem is considered as an illustration, the general case of $N$ regions is detailed in Ref. [19]. The boundary surface between $V_{1}$ and $V_{2}$ is called $S$. The equivalent surface currents are expended in terms of Rao-WiltonGlisson basis functions $\mathbf{f}_{i}^{n}$ [25] forming a triangular mesh approximating $S$ :

$\mathbf{J}_{n}(\mathbf{r})=\sum_{i} \alpha_{i} \mathbf{f}_{i}^{n}(\mathbf{r})$

$\mathbf{M}_{n}(\mathbf{r})=\sum_{i} \beta_{i} \mathbf{f}_{i}^{n}(\mathbf{r})$.

where the index $i$ labels the different edges on $S$. The conservation of current on $S$ requires $\mathbf{J}_{1}=-\mathbf{J}_{2}$ and $\mathbf{M}_{1}=-\mathbf{M}_{2}$, implying $\mathbf{f}_{i}^{1}=-\mathbf{f}_{i}^{2}$ [26]. Similarly to the FEM, the Galerkin method is applied, multiplying Eqs. (8) and (9) by the basis functions and integrating over $S$. We define the sets $\{\alpha\}$ and $\{\beta\}$ of expansion coefficients $\alpha_{i}$ and $\beta_{i}$. The EFIE (8) can be rewritten as a matrix equation:

$\left[\begin{array}{ll}i \omega \mu_{1} \mathbf{D}^{1} & \mathbf{K}^{1} \\ i \omega \mu_{2} \mathbf{D}^{2} & \mathbf{K}^{2}\end{array}\right] \cdot\left[\begin{array}{l}\{\alpha\} \\ \{\beta\}\end{array}\right]=\left[\begin{array}{l}\mathbf{q}^{(E), 1} \\ \mathbf{q}^{(E), 2}\end{array}\right]$,

with submatrices

$D_{i j}^{n}=\int_{S} d S \mathbf{f}_{i}^{n}(\mathbf{r}) \cdot \int_{S} d S^{\prime} \underline{\underline{G}}_{n}\left(\mathbf{r}, \mathbf{r}^{\prime}\right) \cdot \mathbf{f}_{j}^{n}\left(\mathbf{r}^{\prime}\right)$ 
$K_{i j}^{n}=\int_{S} d S \mathbf{f}_{i}^{n}(\mathbf{r}) \cdot \int_{S} d S^{\prime}\left[\nabla^{\prime} \times \underline{\underline{G}}_{n}\left(\mathbf{r}, \mathbf{r}^{\prime}\right)\right] \cdot \mathbf{f}_{j}^{n}\left(\mathbf{r}^{\prime}\right)$,

and

$q_{i}^{(E), n}=\int_{S} d S \mathbf{f}_{i}^{n}(\mathbf{r}) \cdot \mathbf{E}_{n}^{\mathrm{inc}}(\mathbf{r})$.

A similar matrix equation can be found for the MFIE (9):

$$
\left[\begin{array}{ll}
\mathbf{K}^{1} & -i \omega \epsilon_{1} \mathbf{D}^{1} \\
\mathbf{K}^{2} & -i \omega \epsilon_{2} \mathbf{D}^{2}
\end{array}\right] \cdot\left[\begin{array}{l}
\{\alpha\} \\
\{\beta\}
\end{array}\right]=\left[\begin{array}{l}
\mathbf{q}^{(H), 1} \\
\mathbf{q}^{(H), 2}
\end{array}\right]
$$

with

$q_{i}^{(H), n}=-\int_{S} d S \mathbf{f}_{i}^{n}(\mathbf{r}) \cdot \mathbf{H}_{n}^{\mathrm{inc}}(\mathbf{r})$.

In some cases, solving for $\{\alpha\}$ and $\{\beta\}$ with the EFIE or the MFIE do not result in the same values. In fact, especially in resonant conditions, these solutions may also exhibit large errors due to poor testing. The PMCHW formulation [27] which combines EFIE (12) and MFIE (16) to solve them simultaneously has proven to give stable and accurate results:

$$
\begin{aligned}
& {\left[\begin{array}{cc}
i \omega\left(\mu_{1} \mathbf{D}^{1}+\mu_{2} \mathbf{D}^{2}\right) & \mathbf{K}^{1}+\mathbf{K}^{2} \\
\mathbf{K}^{1}+\mathbf{K}^{2} & -i \omega\left(\epsilon_{1} \mathbf{D}^{1}+\epsilon_{2} \mathbf{D}^{2}\right)
\end{array}\right] \cdot\left[\begin{array}{l}
\{\alpha\} \\
\{\beta\}
\end{array}\right]} \\
& \quad=\left[\begin{array}{l}
\mathbf{q}^{(E), 1}+\mathbf{q}^{(E), 2} \\
\mathbf{q}^{(H), 1}+\mathbf{q}^{(H), 2}
\end{array}\right] .
\end{aligned}
$$

Eq. (18) is solved for $\{\alpha\}$ and $\{\beta\}$ to obtain the values of the equivalent surface currents flowing on $S$.

\subsection{Singularity subtraction}

The integral Eqs. (8) and (9) require an evaluation of the periodic dyadic Green's function (7):

$\underline{\underline{G}}_{n}\left(\mathbf{r}, \mathbf{r}^{\prime}\right)=\left(\underline{\underline{1}}+\frac{\nabla \nabla}{k_{n}^{2}}\right) G_{n}\left(\mathbf{r}, \mathbf{r}^{\prime}\right)$,

where

$G_{n}\left(\mathbf{r}, \mathbf{r}^{\prime}\right)=\sum_{\mathbf{t}} \frac{e^{i k_{n}\left|\mathbf{r}-\mathbf{r}^{\prime}-\mathbf{t}\right|}}{4 \pi\left|\mathbf{r}-\mathbf{r}^{\prime}-\mathbf{t}\right|} e^{i \mathbf{k} \cdot \mathbf{t}}$.

The matrix elements in Eqs. (12) and (16) require an integration of the periodic Green's function and its gradient over all possible couples of triangular elements. In the unit cell, the lattice sum (20) has a singularity for $\left|\mathbf{r}-\mathbf{r}^{\prime}\right| \rightarrow 0$. When calculating the matrix elements relative to neighboring triangle areas, the integrand is divergent, yielding inaccurate results in their numerical evaluation. An elegant way to compute these integrals and to improve numerical accuracy is to separate the Green's function into a singular part that can be integrated in a closed form and a smooth slowly varying part that can be accurately integrated numerically:

$G_{n}\left(\mathbf{r}, \mathbf{r}^{\prime}\right)=G_{n}^{s}\left(\mathbf{r}, \mathbf{r}^{\prime}\right)+\frac{1}{4 \pi}\left(\frac{1}{\left|\mathbf{r}-\mathbf{r}^{\prime}\right|}-\frac{k_{n}^{2}\left|\mathbf{r}-\mathbf{r}^{\prime}\right|}{2}\right)$,

where $G_{n}^{s}\left(\mathbf{r}-\mathbf{r}^{\prime}\right)$ is non-singular and differentiable for $\left|\mathbf{r}-\mathbf{r}^{\prime}\right| \rightarrow 0$.

\subsection{Field evaluation}

The surface currents $\mathbf{J}_{n}$ and $\mathbf{M}_{n}$ as solutions of the EFIE and MFIE are not the actual currents flowing on the surfaces $\partial V_{n}$, but they produce the same electromagnetic field inside the regions $V_{n}$. Taking the first choice in Eq. (3) and in its magnetic equivalent, the electric and magnetic fields scattered by the objects are given by

$$
\begin{aligned}
\mathbf{E}_{n}^{\mathrm{scat}}(\mathbf{r})= & -i \omega \mu_{n} \int_{\partial V_{n}} d S^{\prime} \underline{\underline{G}}_{n}\left(\mathbf{r}, \mathbf{r}^{\prime}\right) \cdot \mathbf{J}_{n}\left(\mathbf{r}^{\prime}\right) \\
& -\int_{\partial V_{n}} d S^{\prime}\left[\nabla^{\prime} \times \underline{\underline{G}}_{n}\left(\mathbf{r}, \mathbf{r}^{\prime}\right)\right] \cdot \mathbf{M}_{n}\left(\mathbf{r}^{\prime}\right), \\
\mathbf{H}_{n}^{\text {scat }}(\mathbf{r})= & -i \omega \epsilon_{n} \int_{\partial V_{n}} d S^{\prime} \underline{\underline{G}}_{n}\left(\mathbf{r}, \mathbf{r}^{\prime}\right) \cdot \mathbf{M}_{n}\left(\mathbf{r}^{\prime}\right) \\
& +\int_{\partial V_{n}} d S^{\prime}\left[\nabla^{\prime} \times \underline{\underline{G}}_{n}\left(\mathbf{r}, \mathbf{r}^{\prime}\right)\right] \cdot \mathbf{J}_{n}\left(\mathbf{r}^{\prime}\right) .
\end{aligned}
$$

Hence, the scattered fields can be calculated at any point of space using the Green's tensor. The singularity subtraction technique guarantees a very high accuracy at close vicinity of the scatterer surface.

\section{Numerical example: scattering on arrays of gold bowtie antennas}

This section provides an illustration of the technique in the case of plane-wave excitation of an array of gold bowtie antennas. The antenna is designed in order to ensure the best intensity enhancement in the antenna gap according to Ref. [28] [see Fig. 2(a)]. The discretization with MoM allows a great flexibility in the scatterer's shape: here the corners of the antenna are rounded, which makes it more realistic with respect to the nanofabrication procedures. Experimental data for the frequency-dependant refractive index of gold are 

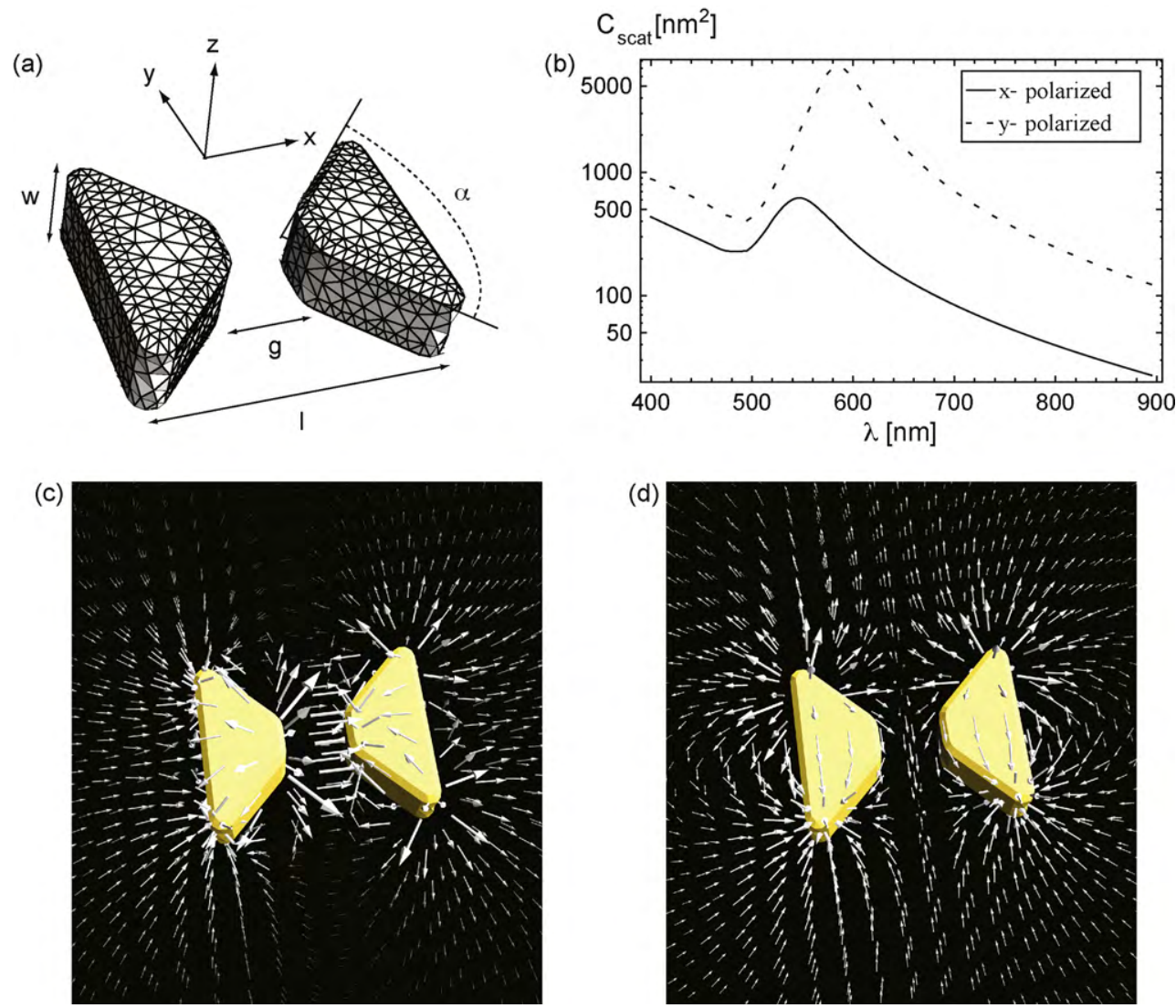

(d)

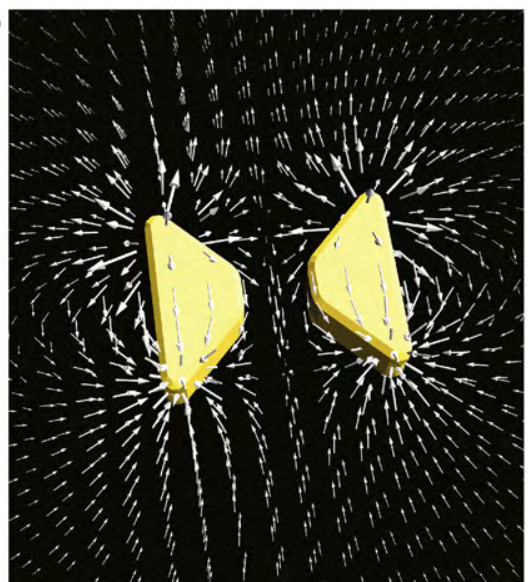

Fig. 2. (a) Design of the bowtie antenna with length $l=110 \mathrm{~nm}$, width $w=30 \mathrm{~nm}$, bow angle $\alpha=90^{\circ}$ and gap size $g=30 \mathrm{~nm}$. The center of the gap is the origin of coordinates. (b) Total scattering cross-section for a $x$-polarized and $y$-polarized plane-wave normal incidence. The real part of the instantaneous electric field is plotted for the following incidence conditions: (c) $x$-polarized at wavelength $550 \mathrm{~nm}$ and (d) $y$-polarized at wavelength $600 \mathrm{~nm}$. Scale: short arrows for low amplitudes, long arrows for high amplitudes.

taken from Ref. [29]. The simulation are performed with 5274 degrees of freedom (i.e. the number of mesh edges times two, since two variables are associated to each edge) ensuring a good convergence of the resonance according to Ref. [17]. The matrix elements are evaluated with a first order Gaussian quadrature [30], resulting in a full matrix. The linear system of equations is solved with a direct solver. Computation time scales with the cube of the number of degrees of freedom. The resonance properties of a single antenna are first investigated, and then compared to the resonances arising in an array.

\subsection{Resonance of a single bowtie antenna}

For the resonance properties of a single bowtie antenna, we used a SIE-based program designed for single object scattering [17].

The scattering cross-section of the antenna has been computed for incidence wavelengths $\lambda$ ranging from
400 to $900 \mathrm{~nm}$ :

$C_{\text {scat }}(\lambda)=R^{2} \int d \sigma \frac{\left|\mathbf{E}_{\lambda}^{\text {scat }}(R, \theta, \phi)\right|^{2}}{\left|\mathbf{E}_{\lambda}^{\text {inc }}\right|^{2}}$,

where $R=5 \mu \mathrm{m}$ is the radius of the surface on which the electric field is sampled (Section 2.4), and $\sigma$ is the solid angle. The problem geometry is given in Fig. 2(a). Fig. 2(b) shows that for a plane-wave polarized in $x$ direction, the scattering cross-section has a resonance with a peak wavelength at $550 \mathrm{~nm}$, corresponding to a mode of the antenna. From Fig. 2(c), we see that for this mode the electric field is confined between the two arms of the antenna. The $y$-polarized mode of Fig. 2(d) has a higher cross section at resonance $[600 \mathrm{~nm}$ from Fig. 2(b)] since the two arms do not interact with each other and behave as two isolated scatterers. The $y$ polarized resonance is red-shifted as compared to the $x$-polarized resonance because the arms are larger along $y$-direction than along $x$-direction. High intensity is 
(a)

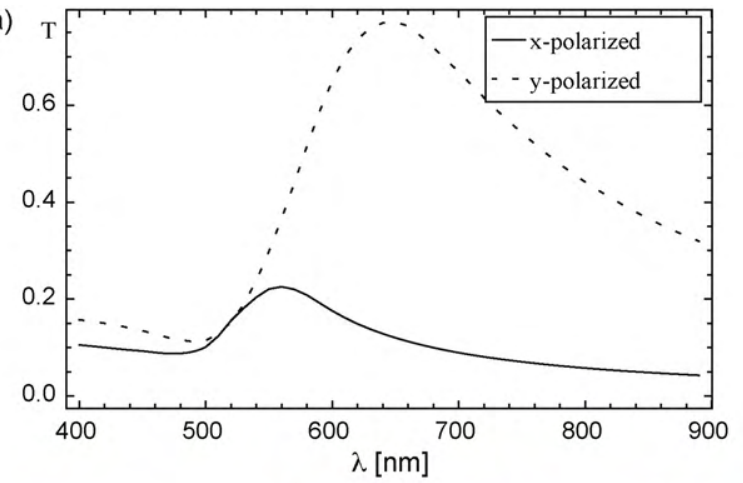

(b)

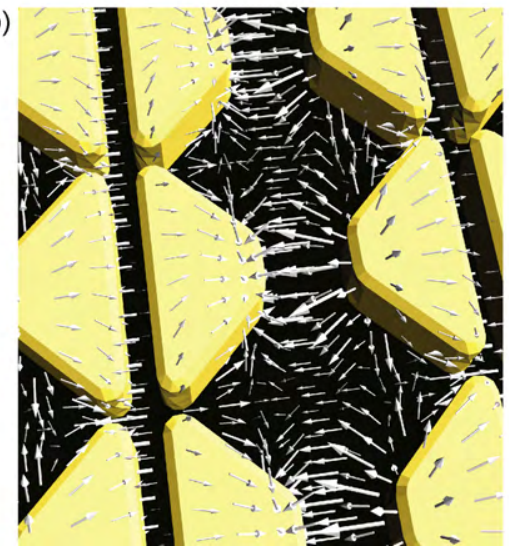

(c)

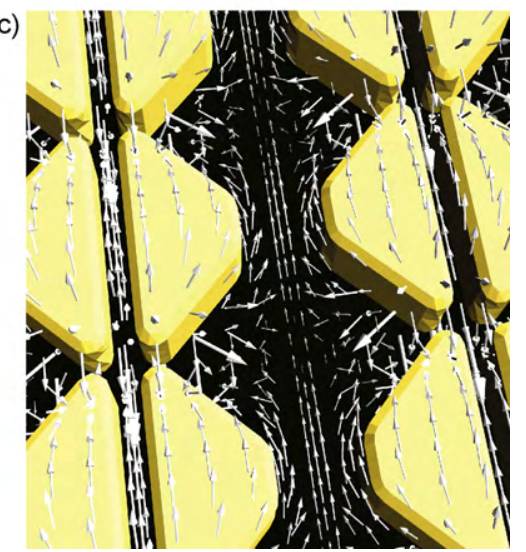

Fig. 3. (a) Transmission through the array of antennas for $x$-polarized and $y$-polarized plane-wave incidence. The real part of the instantaneous electric field is plotted for the following incidence conditions: (b) $x$-polarized at wavelength $560 \mathrm{~nm}$ and (c) $y$-polarized at wavelength $650 \mathrm{~nm}$. Scale: short arrows for low amplitudes, long arrows for high amplitudes.

found at the four corners of the antenna in this case. For the mode at $600 \mathrm{~nm}$, the intensity enhancement is in the order of 80 at position $(50,45,0) \mathrm{nm}$ (i.e. $5 \mathrm{~nm}$ away from a corner of the antenna).

\subsection{Array of bowtie antennas}

A periodicity of $120 \mathrm{~nm}$ along $x$-direction and $90 \mathrm{~nm}$ along $y$-direction is now considered. The transmittance $T(\lambda)$ of the antennas is calculated at $5 \mu \mathrm{m}$ from the array and shown in Fig. 3(a):

$T(\lambda)=\frac{1}{|\Omega|} \int_{\Omega} d^{2} \mathbf{r} \frac{\left|\mathbf{E}_{\lambda}^{\text {scat }}(\mathbf{r})\right|^{2}}{\left|\mathbf{E}_{\lambda}^{\text {inc }}\right|^{2}}$.

For a $x$-polarized incidence, the resonance is slightly red-shifted to $560 \mathrm{~nm}$, and the intensity enhancement in the antenna gap is comparable to Section 3.1. Since the field is mostly confined in each antenna gap, the fact that the antennas are placed in close vicinity to each other does not play a strong role [Fig. 3(b)]. However for a $y$ polarized incidence, the strong coupling between the corners of the antennas results in a red-shift of the resonance from 600 to $650 \mathrm{~nm}$ [Fig. 3(a)]. Higher intensity enhancement is also found in the gap between the antennas [Fig. 3(c)]: it has doubled to 160 at $(50,45,0) \mathrm{nm}$. As can be seen in this example the periodicity strongly affects the optical properties of the plasmonic system. The SIE formulation is able to retrieve both the near-field and far-field properties of such a complex system.

\section{Summary}

The surface integral formulation developed in Ref. [19] has been outlined in the first part of this paper. This method is a flexible, multiscale and accurate tool to simulate light scattering on three-dimensional periodic nanostructures. It reduces the general electromagnetic scattering problem to a discretizated model using the Method of Moments on the surface of the scatterers in the unit cell. The study of the resonances of an array of bowtie antennas has been used to illustrate the applicability of the method to plasmonic systems. 
When placed into arrays, the bowtie antennas resonances are strongly affected when compared to those of an individual antenna. Using the surface integral formulation, we have been able to investigate both near-field and far-field properties of these resonances with a high level of accuracy.

\section{Acknowledgments}

It is a pleasure to acknowledge stimulating discussions with M. Schnieper and A. Stuck. The authors warmly thank Pascal Bérard for providing the 3D data visualization. This work was supported by CCMXSPERU.

\section{References}

[1] J.P. Kottmann, O.J.F. Martin, D.R. Smith, S. Schultz, Plasmon resonances of silver nanowires with a nonregular cross-section, Physical Review B 64 (2001) 235402.

[2] V.M. Shalaev, W.S. Cai, U.K. Chettiar, H.K. Yuan, A.K. Sarychev, V.P. Drachev, A.V. Kildishev, Negative index of refraction in optical metamaterials, Optics Letters 30 (2005) 3356-3358.

[3] S.C. Kitson, W.L. Barnes, J.R. Sambles, Full photonic band-gap for surface modes in the visible, Physical Review Letters 77 (1996) 2670.

[4] M. Sarrazin, J.P. Vigneron, J.M. Vigoureux, Role of Wood anomalies in optical properties of thin metallic films with a bidimensional array of subwavelength holes, Physical Review B 67 (2003) 085415.

[5] C. Genet, M.P. van Exter, J.P. Woerdman, Fano-type interpretation of red shifts and red tails in hole array transmission spectra, Optics Communications 225 (2003) 331.

[6] W.L. Barnes, A. Dereux, T.W. Ebbesen, Surface plasmon subwavelength optics, Nature 424 (2003) 824-830.

[7] K.B. Crozier, E. Togan, E. Simsek, T. Yang, Experimental measurement of the dispersion relations of the surface plasmon modes of metal nanoparticle chains, Optics Express 15 (2007) 17482.

[8] M. Hammer, Hybrid Analytical/numerical coupled-mode Modeling of guided-wave devices, Journal of Lightwave Technology 25 (2007) 2287.

[9] K. Yee, Numerical solution of initial boundary value problems involving Maxwell's equations in isotropic media, IEEE Transactions on Antennas and Propagation 14 (1966) 302-307.

[10] K. Sakoda, Optical Properties of Photonic Crystals, Springer, Berlin, 2005

[11] J. Jin, Finite Element Method in Electromagnetics, Wiley, 2002.

[12] D. Lockau, L. Zschiedrich, S. Burger, Accurate simulation of light transmission through subwavelength apertures in metal films, Journal of Optics A-Pure and Applied Optics 11 (2009) 114013.

[13] H. Fischer, A. Nesci, G. Leveque, O.J.F. Martin, Characterization of the polarization sensitivity anisotropy of a near-field probe using phase measurements, Journal of Microscopy-Oxford 230 (2008) 27.
[14] P.C. Chaumet, A. Rahmani, G.W. Bryant, Generalization of the coupled dipole method to periodic structures, Physical Review B 67 (2003) 165404.

[15] B.T. Draine, P.J. Flatau, Discrete-dipole approximation for periodic targets: theory and tests, Journal of the Optical Society of America A-Optics Image Science and Vision 25 (2008) 2693.

[16] F.J. García de Abajo, A. Howie, Retarded field calculation of electron energy loss in inhomogeneous dielectrics, Physical Review B 65 (11) (2002) 115418.

[17] A.M. Kern, O.J.F. Martin, Surface integral formulation for 3D simulations of plasmonic and high permittivity nanostructures, Journal of the Optical Society of America A-Optics Image Science and Vision 26 (2009) 732-740.

[18] T. Eibert, J. Volakis, D. Wilton, D. Jackson, Hybrid FE/BI modeling of 3D doubly periodic structures utilizing triangular prismatic elements and an MPIE formulation accelerated by the Ewald transformation, IEEE Transactions on Antennas and Propagation 47 (5) (1999) 843-850.

[19] B. Gallinet, O.J.F. Martin, Electromagnetic scattering on periodic nanostructures: modeling with a surface integral formulation, in preparation.

[20] N. Marly, B. Baekelandt, D. De Zutter, H. Pues, Integral equation modeling of the scattering and absorption of multilayered doubly periodic lossy structures, IEEE Transactions on Antennas and Propagation 43 (11) (1995) 1281-1287.

[21] L. Trintinalia, H. Ling, Integral equation modeling of multilayered doubly periodic lossy structures using periodic boundary condition and a connection scheme, IEEE Transactions on Antennas and Propagation 52 (9) (2004) 2253-2261.

[22] I. Stevanovic, P. Crespo-Valero, K. Blagovic, F. Bongard, J.R. Mosig, Integral-equation analysis of 3D metallic objects arranged in $2 \mathrm{D}$ lattices using the Ewald transformation, IEEE Transactions on Microwave Theory and Techniques 54 (2006) 3688-3697.

[23] F. Capolino, D.R. Wilton, W.A. Johnson, Efficient computation of the 3D Green's function for the Helmholtz operator for a linear array of point sources using the Ewald method, Journal of Computational Physics 223 (2007) 250-261.

[24] I. Stevanoviae, J.R. Mosig, Periodic Green's function for skewed 3D lattices using the Ewald transformation, Microwave and Optical Technology Letters 49 (2007) 1353-1357.

[25] S. Rao, D. Wilton, A. Glisson, Electromagnetic scattering by surfaces of arbitrary shape, IEEE Transactions on Antennas and Propagation 30 (1982) 409-418.

[26] P. Ylä-Oijala, M. Taskinen, J. Sarvas, Surface integral equation method for general composite metallic and dielectric structures with junctions, Progress in Electromagnetics Research-PIER 52 (2005) 81-108.

[27] T.K. Wu, L.L. Tsai, Scattering from arbitrarily shaped lossy dielectric bodies of revolution, Radio Science 12 (1977) 709 718.

[28] H. Fischer, O.J.F. Martin, Engineering the optical response of plasmonic nanoantennas, Optics Express 16 (2008) 9144.

[29] P.B. Johnson, R.W. Christy, Optical-constants of noble-metals, Physical Review B 6 (1972) 4370.

[30] G.R. Cowper, Gaussian quadrature formulas for triangles, International Journal for Numerical Methods in Engineering 7 (1973) 405-408. 